\title{
Quantifying water absorption of hygrothermally aged epoxies with terahertz time-domain spectroscopy
}

\author{
Hungyen Lin ${ }^{1}$, Benjamin P. Russell ${ }^{2}$, Prince Bawuah ${ }^{3}$ and J. Axel Zeitler ${ }^{2}$ \\ ${ }^{1}$ Department of Engineering, Lancaster University, Lancaster LA1 4YW, United Kingdom \\ ${ }^{2}$ Experimental Materials Science, Hexcel Composites Ltd., Duxford CB22 4QB, United Kingdom \\ ${ }^{3}$ Department of Chemical Engineering and Biotechnology, University of Cambridge CB3 0AS, United Kingdom
}

\begin{abstract}
We demonstrate how terahertz time-domain spectroscopy (THz-TDS) can be used to quantify water absorption in hygrothermally aged simple and commercial epoxy systems supported by conventional gravimetric analysis.
\end{abstract}

\section{INTRODUCTION}

$\mathrm{E}$ POXIES represent an interesting class of materials containing reactive monomers that cross-link either with themselves or with hardeners such as amines in the curing process. As such, they have found applications as encapsulations in the electronics industry, adhesives in structural bonding, anticorrosive protective coatings on metal structures, and in the creation of composites. The advent of terahertz sensing has also opened up many exciting industrial applications [1-6] and for studying technical polymers [7, 8]. Even though epoxies have been studied by THz-TDS [9], there is no report on their water uptake properties. In this work, we demonstrate how THz-TDS can be used to quantify water absorption in hygrothermally aged epoxy systems, for both simple and commercial systems, supported by conventional gravimetric analysis [10].

\section{METHODS}

A total of five epoxy systems were prepared: two commercial systems with the names 8552 and RMT6, and three simple systems of the same epoxy (Epikote 828) and amine (Jeffamine T403) at 3 different stoichiometries. The hygrothermal conditions were performed by immersing the samples in distilled water in a beaker at a temperature of $95{ }^{\circ} \mathrm{C}$ (Fig. 1). At each time increment, the samples were removed, blotted dry, characterised in terms of mass, thickness and THz-TDS, before returning back to the conditioning chamber. The duration of the ageing experiment was $80 \mathrm{~h}$ in total. We performed transmission $\mathrm{THz}$ spectroscopy using a commercial THz-TDS setup (Terapulse 4000, TeraView Ltd., Cambridge, U.K.). Frequency-dependent optical constants can then be calculated from the measurements and converted to complex relative permittivity. The complex permittivity of aged epoxy $\tilde{\varepsilon}_{k}(v)$ can be described using the Landau, Lifshitz, Looyenga (LLL) model [10] as

$$
\sqrt[3]{\tilde{\varepsilon}_{k}(v)}=f_{\mathrm{BW}, k} \sqrt[3]{\tilde{\varepsilon}_{\mathrm{BW}}(v)}+\left(1-f_{\mathrm{BW}, k}\right) \sqrt[3]{\tilde{\varepsilon}_{\mathrm{D}}(v)}
$$

where $\tilde{\varepsilon}_{\mathrm{BW}}(v)$ is the complex permittivity of bound water, $\tilde{\varepsilon}_{\mathrm{D}}(v)$ is the complex permittivity of the dry epoxy, volumetric fractions $f_{\mathrm{BW}, k}$ and $1-f_{\mathrm{BW}, k}$ for a two component mixture. The volumetric fraction $f_{\mathrm{BW}, k}$ is determined by $f_{\mathrm{BW}, k}=\frac{m_{k}-m_{0}}{\rho A d_{k}}$ where $m_{k}$ is the mass of aged epoxy at time $k, m_{0}$ is the mass of dry epoxy before conditioning, $\rho$ is the density of water taken as $1 \mathrm{~g} . \mathrm{cm}^{-3}$, and $A$ is the sample area.
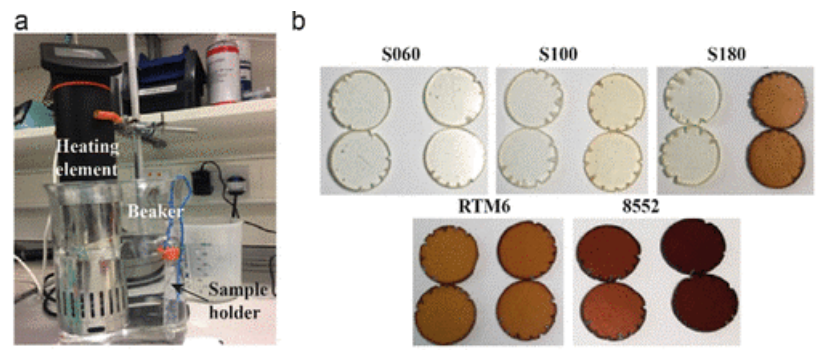

Fig. 1. a) Water chamber setup to age the epoxies. b) Epoxy specimens before and after conditioning.

\section{RESULTS}

Fig. $1 \mathrm{~b}$ shows that the samples all underwent distinct irreversible yellowing with S180 being affected the most affected and S060 the least. Yellowing in bulk is a well-known phenomenon in aliphatic amine cured epoxy systems, and relates to various oxidation processes in which $\mathrm{N}-\mathrm{H} 2$ groups participate [11]. The extracted frequency dependent refractive indices and absorption coefficients of the samples at different time increments are shown in Fig. 2
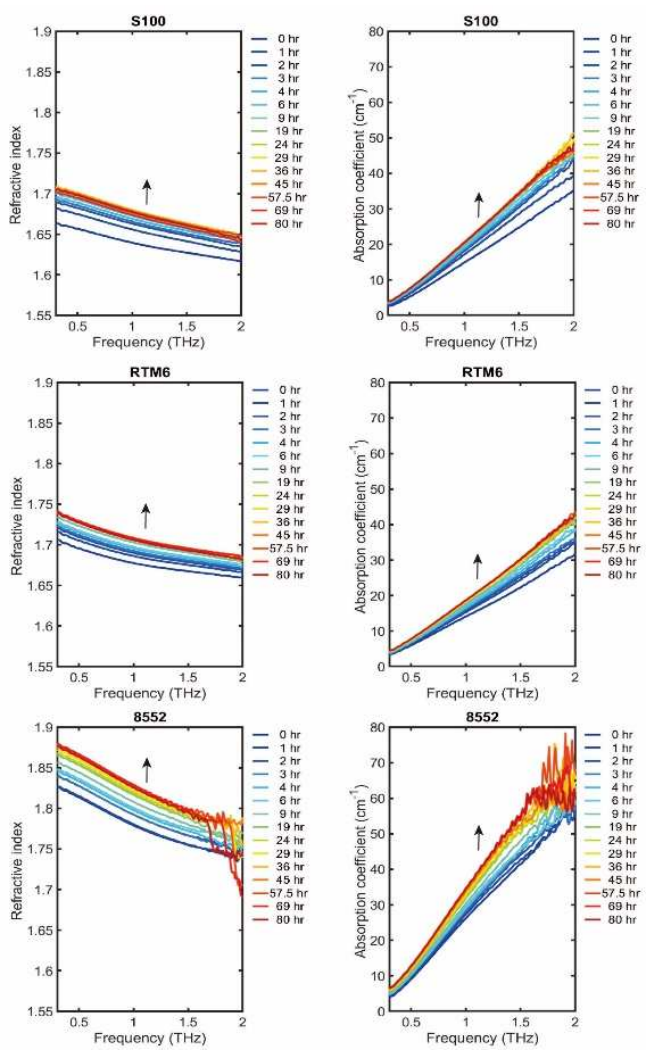

Fig. 2. Frequency dependent optical constants of the specimens at different water ageing time. 
In order to compare against the conventional gravimetric analysis, Fig 3 shows the optical constants at $1 \mathrm{THz}$ as a function of water ageing time where a linear correlation can be observed.
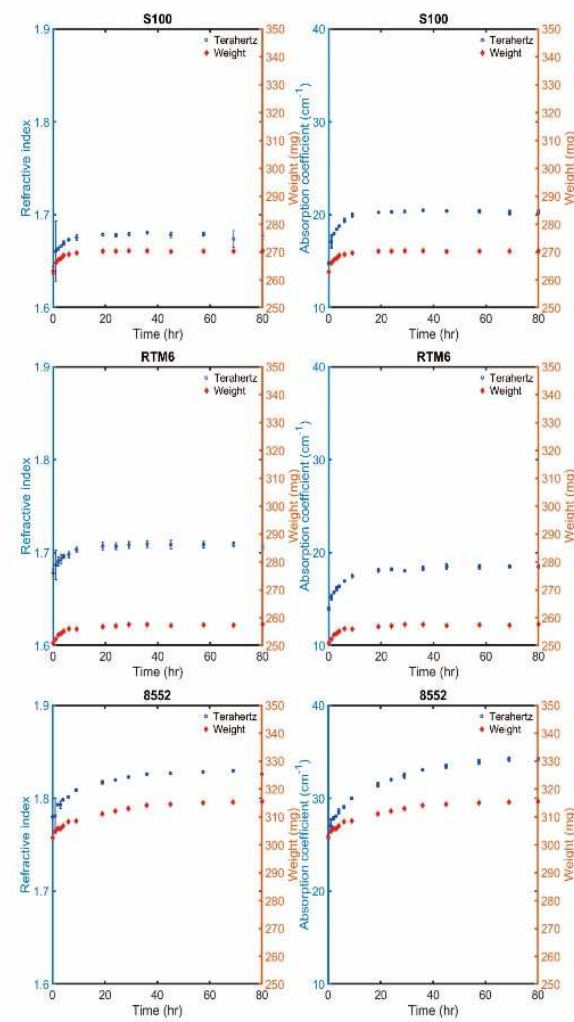

Fig. 3. Optical constants at $1 \mathrm{THz}$ with gravimetric analysis as a function of water uptake.
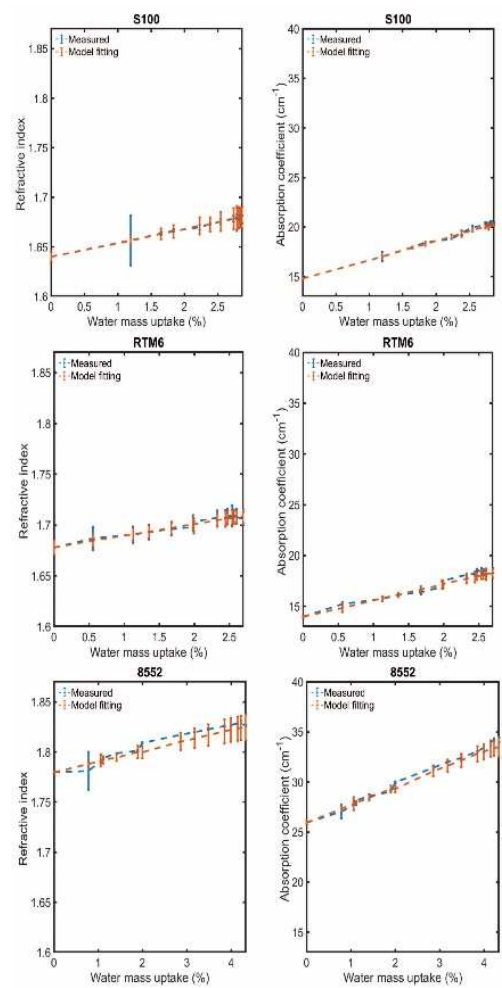

Fig. 4. Optical constants at $1 \mathrm{THz}$ from measurement and estimated using the LLL model as a function of water uptake. Lines plotted to guide the eye.
Finally, Fig. 4 compares the optical constants from the measurements and from the LLL model as a function of water uptake where good agreement is observed.

\section{SUMMARY}

We have demonstrated how THz-TDS could be used to resolve water absorption during a hygrothermal ageing process in different epoxy systems benchmarked by conventional gravimetric analysis. Using the LLL model, water uptake can be quantified at sufficient fidelity. This therefore suggest that THz-TDS could be used to complement existing techniquesin accelerated degradation testing.

\section{ACKNOWLEDGEMENT}

HL acknowledges financial support EPSRC (Grant No. EP/R019460/1 and Teranet Seedcorn Fund EP/M00306X/1).

\section{REFERENCES}

[1]. A. K. Vynckier, et al.. "Calendering as a direct shaping tool for the continuous production of fixed-dose combination products via co-extrusion. Eur. J. Pharm. Biopharm. 96, 125-131, 2015.

[2]. D. Alves-Lima, et al., "Review of Terahertz Pulsed Imaging for Pharmaceutical Film Coating Analysis," Sensors, 20, 1441, 2020.

[3]. D. F. Alves-Lima, et al., "Quantitative video-rate hydration imaging of Nafion proton exchange membranes with terahertz radiation," J. Power Sources, 450, 227665, 2020.

[4]. H. Lin, et al., "Review of THz near-field methods," Proceedings of the Smart Structures, Devices, and Systems III, Adelaide, Australia, 10-13 December 2006; SPIE: Bellingham, WA, USA, 2006.

[5]. D. Y. S. Chau, et al., "A. determination of water content in dehydrated mammalian cells using terahertz pulsed imaging: a feasibility study," Curr. Pharm. Biotechnol. 17, 200-207, 2015.

[6]. H. Lin, et al., "Gas recognition with terahertz time-domain spectroscopy and spectral catalog: A preliminary study," Proc. SPIE Terahertz Photonics, 6840, 68400X, 2007.

[7] S. Wietzke et al., "Terahertz spectroscopy on polymers: A review of morphological studies," Journal of Molecular Structure, vol. 1006, 41-51, 2011. [8] C. Jordens, et al., "Investigation of the water absorption in polyamide and wood plastic composite by terahertz time-domain spectroscopy," Polym. Test., vol. 29, 209-215, 2010.

[9] E. Stübling et al., "THz properties of adhesives," J. Infrared, Millimeter, Terahertz Waves, vol. 39, 586- 593, 2018.

[10]. H. Lin et al., "Sensing Water Absorption in Hygrothermally Aged Epoxies with Terahertz Time-Domain Spectroscopy," Anal. Chem., vol. 93, 2449-2455, 2021.

[11]. H. Looyenga, "Dielectric Constants of Heterogeneous Mixtures," Physica, vol. 31, 401-406, 1965.

[12] A. E. Krauklis and A.T. Echtermeyer, Mechanism of Yellowing: Carbonyl Formation during Hygrothermal Aging in a Common Amine Epoxy. Polymers, vol. 10, 1017, 2018. 